\title{
High Volume Portal Usage Impacts Practice Resources
}

\author{
Augustine Chavez, MD, Jesse Bracamonte, DO, Molly Kresin, DO, \\ Michael Yardley, MBA, and Michael Grover, DO
}

Patient portals have both patient-centered benefits and substantial impacts on practices. Successful implementation of a patient portal can be achieved with a comprehensive team approach. Increased portal usage results in high-volume patient secure messaging. We describe the impact of high patient message volume on our fee-for-service academic family medicine practice. Practice adaptations are necessary to manage volume, length, and complexity of messages. Dedicated staff with specialized training to handle patient messages and ample protected time for providers are important to minimize clerical burden associated with patient portal use. ( $\mathrm{J}$ Am Board Fam Med 2020;33:452-455.)

Keywords: Fee-for-Service Plans, Patient-Centered Care, Patient Portals

\section{Background}

Patient portals are associated with several patientcentered benefits. Portal usage can improve management of chronic disease, ${ }^{1,2}$ enhance treatment compliance, and improve patient engagement and patient-provider communication. ${ }^{3,4}$ Patients enjoy communicating with their providers remotely.,6 The longer patients participate in portal usage, the more likely they are to prefer the portal for communication. $^{7}$

Despite multiple benefits, rates of portal participation remain low, ranging between $10 \%$ and $52 \%$, with most groups reporting participation of only $30 \% .^{2,8,9}$ These low rates have prompted articles encouraging and describing strategies to increase portal implementation. ${ }^{10}$

Consequences of robust patient portal implementation include impacts on provider and practice workloads. $^{6,8,11}$ Clerical burdens associated with electronic health record (EHR) use, including excessive time spent after hours on inbox management, are linked to provider burnout. ${ }^{12}$ Of particular

This article was externally peer reviewed.

Submitted 29 October 2019; revised 15 January 2020; accepted 21 January 2020.

From Mayo Clinic Arizona, Scottsdale (AC, JB, MK, MG); Mayo Clinic Minnesota, Rochester (MY).

Funding: None.

Conflicts of Interest: None.

Corresponding author: Augustine Chavez, MD, Mayo Clinic Arizona, 13737 N 92nd St, Scottsdale, AZ 85260 (E-mail: Chavez.Augustine@mayo.edu). concern are patient-generated secure messages. Patients initiate portal messages more frequently than providers. ${ }^{13}$ Increased patient-initiated portal messages do not result in reduced phone messages or office visits. $^{14,15}$

Appreciating the patient-centered benefits of portal usage while acknowledging the substantial impact on provider and practice workflows is critical for successful management of a patient portal. This article describes our experience in successfully promoting high rates of patient portal engagement and the subsequent challenges to our practice.

\section{Implementation}

Our practice is a fee-for-service academic family medicine department composed of salaried physicians and nurse practitioners in Arizona. Eightyfour percent of our 23,000 empaneled patients had portal accounts by 2018. Forty percent access their portal, and $10.5 \%$ send a message monthly. Between 2015 and 2018, we saw a 275\% increase in unique patients sending portal messages, with total volume reaching 3832 per month (Figure 1).

Consistent with prior documented successful efforts at increasing portal uptake, ${ }^{9}$ we use a comprehensive team approach to enrollment. Call center staff offer to enroll patients during each call, online portal signup sheets are available at check-in and check-out desks, and online portal signup sheets are mailed to all new patients. Nursing staff 
Figure 1. Average monthly inbox messages.

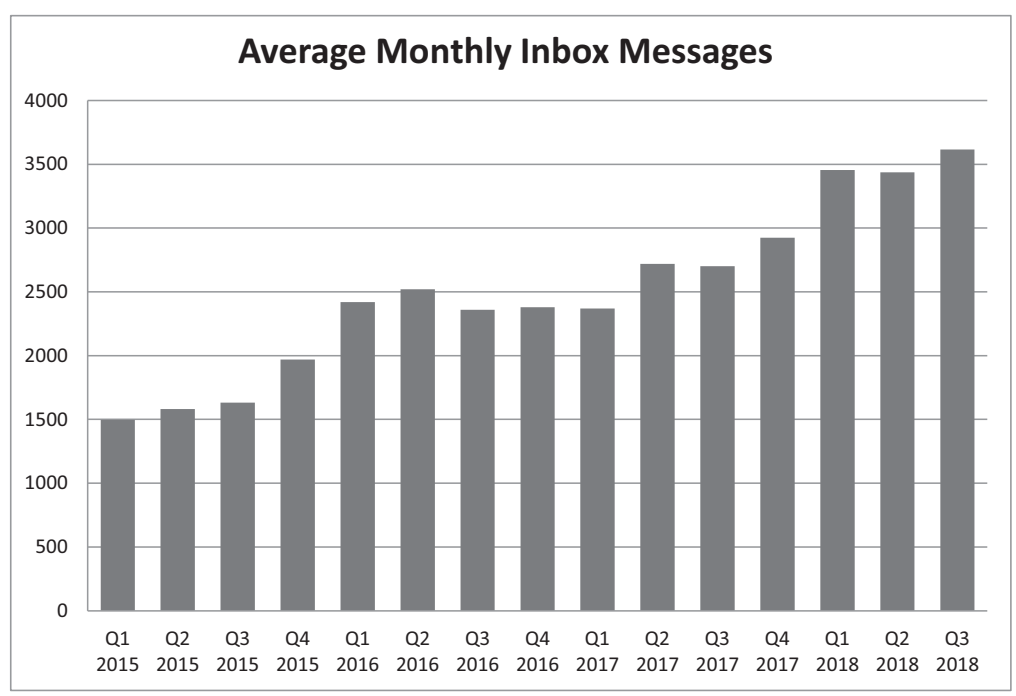

ensures patients have portal access and, if not, asks patients to enroll. To establish patient expectations, a card providing information on when and under what circumstances patients should use the portal is distributed to patients in their appointment packet and at the check-out desk.

Our providers experienced a marked increase in asynchronous patient work with implementation of the patient portal. These clerical burdens were reflected in annual staff surveys demonstrating decreasing workplace satisfaction, increased burnout, and dissatisfaction with use of the EHR. In response, institutional leadership granted providers $10 \%$ of their full-time equivalent (FTE) each day as protected time for management of inbox tasks. In addition, when providers are away for vacation or education, their inbox is managed by a colleague proxy who is assigned an additional hour a day for inbox management.

Our workflows use pools for triage of messages. All new patient-generated messages are first received by front desk staff, where they are resolved (eg, if an appointment request) or forwarded to the appropriate clinical or nonclinical pool (eg, billing or nursing). Clinical messages are sent to team pools managed by nurses or medical assistants, where they can be resolved or supplemented with additional clinical information and/or pended orders. Messages requiring provider action are forwarded to the patient's primary care physician with the added clinical information and orders included. Based on daily staffing availability, the nursing message pool could be assigned to a single person or, more commonly, to the entire nursing group.

\section{Impact}

Our providers receive a daily average of 41.3 messages, of which $16 \%$ are patient-generated secure messages. The burden of messages is influenced by their length, content, urgency, and the degree of value added by others before being sent to the provider.

Our practice receives some patient-generated messages exceeding 5000 characters in length, often seeking advice for new or undifferentiated problems. Although the message length may include valuable history related to the concern, the size of the message alone is commonly cited as a source of provider stress and an example of portal misuse. One proposed solution to excessively long messages is limitations on character counts. However, character count limits may lead patients to send multiple separate messages to convey their concerns.

Sending multiple messages impacts efficiency and creates confusion in our practice. When a patient sends multiple messages, this creates multiple separate encounters in the EHR. The thread of messages should be linked together, but each individual message may be handled by a different nurse, resulting in several incomplete messages rather than one comprehensive product.

Long, fragmented message threads create particular concern for clinically important or urgent messages. Though some data suggest that very few 
Figure 2. Inbox management burden. Abbreviations: FTE, full-time equivalent.

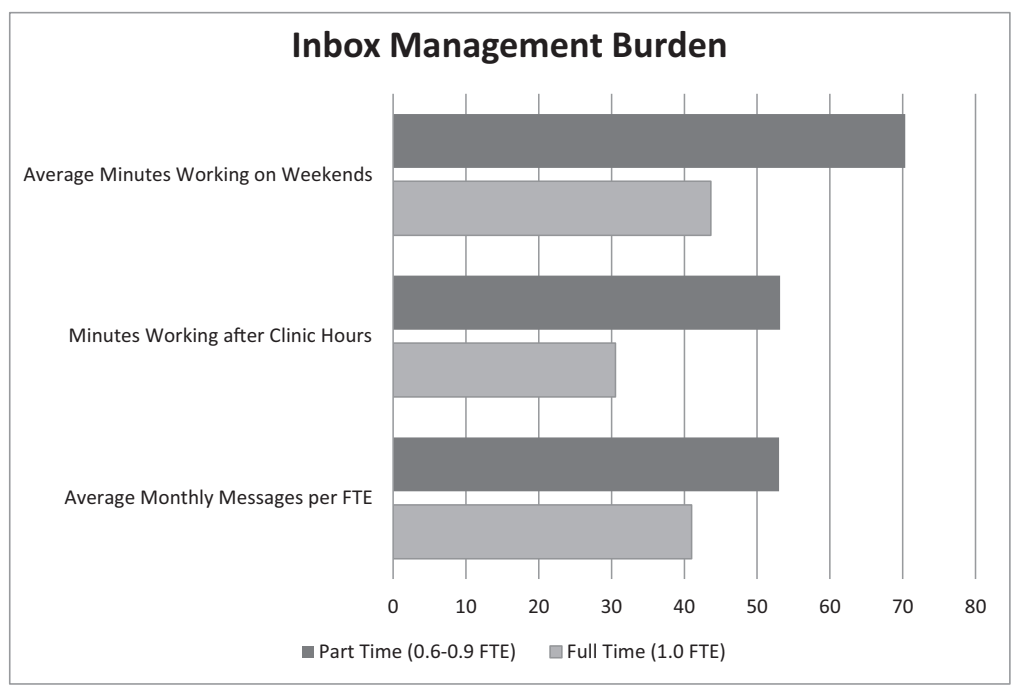

patient-generated messages are about new symptoms or are clinically urgent, ${ }^{16}$ other data demonstrate that $63 \%$ of messages require clinical interpretation ${ }^{17}$ and, therefore, the input of a provider. Our workflow relies on intermediate steps from other team members to triage and supplement the information in a message. These processes take time. Slow responses can create additional issues for the practice.

A disclaimer is displayed to patients sending portal messages advising them to call 911 for emergencies or urgent medical questions and stating responses can be expected within 2 business days. Despite clearly stated expectations for turnaround time, our experience confirms prior published worries that patients expect rapid, 24-hour-a-day, 7-days-a-week responses. ${ }^{16}$ In a single day, we have noted patients sending multiple messages to their provider, messaging multiple different providers, and placing a telephone call all in regard to the same issue. This duplication of messages not only adds to the total volume of messages but also the stress and frustration providers feel when managing their inbox.

Allowing adequate time for nurses to review messages, determine if additional information is necessary, and acquire it before sending messages to providers is important. Ideally, appropriate orders for prescription renewals, lab or imaging tests, and follow-up visits would be pended for provider signature. However, our providers perceive high variability in the completeness of messages they receive.

This variability stems from both individual and systems characteristics. Individual nurses or medical assistants have variable understanding of what information is important to add to a message based on their level of education and prior job experiences. Training initiatives can lessen variability and improve message quality, but turnover and use of supplemental staff makes consistency a challenge. A team member trained to the highest level of his or her license dedicated to addressing clinical messages might improve message quality; however, this would require hiring additional staff.

Provider message burdens could be reduced if some messages were prevented from ever arriving in the in-basket. Staff could use protocols to resolve low-risk clinical requests including signing standing orders, scheduling appointments, or prescribing low-risk medications. However, state-defined scope of practice and institutional definitions of roles and responsibilities pose barriers to implementation of these protocols, ultimately meaning final resolution of most messages requires a provider's involvement.

Our current allotment of protected time for inbox management has been overwhelmed by the high volume of messages, which are often long, disjointed, urgent, and incomplete. Internal EHR data reveal that our providers work on their inbox an average of 39 minutes outside of clinic hours on work days and up to 180 minutes on days off.

Paradoxically, part-time providers in our practice have proportionately higher message volumes and spend more time after hours and on nonworking days than full-time providers (Figure 2) despite panel sizes being adjusted to FTE. One possible explanation is that patients send more complex portal 
messages in response to decreased provider availability for appointments. These complex messages in turn require more provider time and effort. Appointment availability is reduced in ways besides FTE reductions. In our practice, complex visits are assigned longer appointment times. Test results are communicated through the portal, eliminating simple "results review" visits. Consequently, we have seen an increase in long compared with short appointments reducing total appointment availability. Similarly, our protected in-box management time also reduces appointment access. This has prompted some providers to overbook patients into that dedicated time, thereby shifting inbox management to after hours and off days.

\section{Conclusions}

Efforts to increase patient portal engagement resulted in marked increased patient-generated portal messages that can be long, fragmented, and incomplete. These messages create added work but do not decrease phone calls or office visits. Additional, dedicated nurses to triage, supplement, and resolve patient messages could improve quality and reduce number of messages arriving in provider inboxes. Providers require protected time to manage inbox messages. Our current allotment is inadequate to prevent after-hours and weekend inbox management. The work of primary care providers is shifting from face-to-face interactions to virtual patient management, including through patient portals. Our experiences demonstrate that provision of adequate time, dedicated staff with enhanced responsibilities, and standardized workflows are essential to optimal asynchronous patient care. Beginning in 2020, Current Procedural Terminology codes 99421-43 will reimburse for some electronic services. Advocacy for additional resources is necessary for practices to succeed in this evolving care model.

To see this article online, please go to: bttp://jabfm.org/content/ 33/3/452.full.

\section{References}

1. Manard W, Scherrer JF, Salas J, et al. Patient portal use and blood pressure control in newly diagnosed hypertension. J Am Board Fam Med 2016;29:452-9.

2. Devkota B, Salas J, Sayavong S, et al. Use of an online patient portal and glucose control in primary care patients with diabetes. Popul Health Manag 2016;19:125-31.
3. Bush RA, Connelly CD, Pérez A, et al. Physician perception of the role of the patient portal in pediatric health. J Ambul Care Manage 2017;40:238-45.

4. Baldwin JL, Singh H, Sittig DF, et al. Patient portals and health apps: pitfalls, promises, and what one might learn from the other. Healthc (Amst) 2017;5:81-5.

5. Sedlander E, Barboza KC, Jensen A, et al. Veterans' preferences for remote management of chronic conditions. Telemed J E Health 2018;24:229-35.

6. Hoonakker PLT, Carayon P, Cartmill RS. The impact of secure messaging on workflow in primary care: results of a multiple-case, multiple-method study. Int J Med Inform 2017;100:63-76.

7. Choudhry A, Hong J, Chong K, et al. Patients' preferences for biopsy result notification in an era of electronic messaging methods. JAMA Dermatol 2015;151:513-21.

8. Miller DP, Latulipe C, Melius KA, Jr, et al. Primary care providers' views of patient portals: interview study of perceived benefits and consequences. J Med Internet Res 2016;18:e8.

9. Krist AH, Woolf SH, Bello GA, et al. Engaging primary care patients to use a patient-centered personal health record. Ann Fam Med 2014;12:418-26.

10. Twiddy D. Removing six key barriers to online portal use. Fam Pract Manag 2015;22:26-31.

11. Gerber DE, Beg MS, Duncan T, et al. Oncology nursing perceptions of patient electronic portal use: a qualitative analysis. Oncol Nurs Forum 2017;44:165-70.

12. Shanafelt TD, Dyrbye LN, Sinsky C, et al. Relationship between clerical burden and characteristics of the electronic environment with physician burnout and professional satisfaction. Mayo Clin Proc 2016;91:836-48.

13. Shimada SL, Petrakis BA, Rothendler JA, et al. An analysis of patient-provider secure messaging at two Veterans Health Administration medical centers: message content and resolution through secure messaging. J Am Med Inform Assoc 2017;24:942-9.

14. Dexter EN, Fields S, Rdesinski RE, et al. Patientprovider communication: does electronic messaging reduce incoming telephone calls? J Am Board Fam Med 2016;29:613-9.

15. North F, Crane SJ, Chaudhry R, et al. Impact of patient portal secure messages and electronic visits on adult primary care office visits. Telemed J E Health 2014;20:192-8.

16. North F, Crane SJ, Stroebel RJ, et al. Patient-generated secure messages and $\mathrm{eVisits}$ on a patient portal: are patients at risk? J Am Med Inform Assoc 2013;20:1143-9.

17. Garrido T, Meng D, Wang JJ, et al. Secure e-mailing between physicians and patients: transformational change in ambulatory care. J Ambul Care Manage 2014;37:211-8. 